\title{
Determinantes en la Estrategia de Internacionalización para las Pymes: el Caso de Chile
}

\author{
Carlos Poblete', J.E.Amorós
}

\begin{abstract}
The rapid internationalization of business can be a very important factor in their development, so that they can then become established companies that create more jobs and develop innovation. Through the observation of a group of Chilean SMEs, this paper aims to verify compliance with the accelerated internationalization theory developed by Oviatt and McDougall (1994) for Chile. Analyzing a sample of II2 SMEs it could be shown that in the case of Chile, there are factors proposed in the literature, such as support networks, the size of the company and the technological level of the industry, that not necessarily accelerate the internationalization strategy. Moreover, the impact of psychic distance and previous experience are be factors to accelerate the search for international markets. From these results, it is suggested that policy makers should focus more on strengthening the human capital of individual entrepreneurs, instead of institutional aspects.

La rápida internacionalización de las empresas puede ser un factor muy importante para su desarrollo, de manera que éstas luego puedan convertirse en empresas consolidadas que generen más empleo y desarrollen la innovación. A través de la observación de un conjunto de pymes chilenas, en este trabajo se pretende verificar el cumplimiento de la teoría de internacionalización acelerada desarrollada por Oviatt y McDougall (1994) para el caso chileno. Analizando una muestra de II2 pymes exportadoras se pudo demostrar que para el caso de Chile existen factores propuestos por la literatura, como las redes de apoyo, el tamaño de la empresa y el nivel tecnológico de la industria, que no necesariamente aceleran la estrategia de internacionalización. Por otra parte, el impacto de las distancias psíquicas y la experiencia previa resultan ser factores determinantes para acelerar la búsqueda de mercados internacionales. A partir de los resultados, se sugiere que la política pública deba enfocarse más en fortalecer el capital humano de los individuos emprendedores, que en netamente aspectos institucionales.
\end{abstract}

Keywords: internationalization strategy; born globals; international new ventures; international business; estrategia de internacionalización; born globals; international new ventures; negocios internacionales.

Este trabajo se ha realizado bajo el apoyo del Proyecto Fondecyt $N^{\circ}$ I 100819 "Born Globals y Micromultinacionales: Un Analisis InterRegional del Emprendimiento Internacional".

'Av. La Plaza, 680, Las Condes. Santiago, Chile. Phone: 02 3279158. email: capobletec@udd.cl.

ISSN: 07 I8-2724. (http://www.jotmi.org)

Journal of Technology Management \& Innovation @ Universidad Alberto Hurtado, Facultad de Economía y Negocios. 


\section{Introducción}

Existen diversas teorías que intentan explicar los determinantes de la internacionalización de las empresas hacia otros mercados. Principalmente existen dos grupos de teorías que pretenden explicar el comportamiento exportador de las pequeñas y medianas empresas.

El primer grupo hace referencia a un proceso gradual de internacionalización, en la que se cumplen etapas. Según este enfoque existe un procedimiento de internacionalización en la que las empresas poco a poco van comprometiendo, de forma gradual, sus recursos y esfuerzos a hacia este objetivo. Esta teoría fue desarrollada por autores como Johanson y Wiedersheim (1975), Vahlne y Nordström (1993), Johanson y Vahlne (1990), entre otros.

El segundo grupo se refiere a procesos acelerados de internacionalización. Estas teorías explican que es posible encontrar empresas que desde el inicio se enfocan en la internacionalización del negocio. Las empresas que siguen el patrón de internacionalización acelerado comienzan con un énfasis en el crecimiento a través de ventas en los mercados extranjeros. Este tipo de empresas, a diferencia de lo que señala el modelo gradual, no esperan ganar experiencia en el mercado local, y luego en mercados similares para finalmente expandirse a otros países y/o continentes. Por el contrario, desde prácticamente su año de fundación comienzan exportando a diferentes partes del mundo (Madsen y Servais, 1997).

Dentro de esta corriente existe una gran variedad de denominaciones que caracterizarían el mismo fenómeno, aalgunas de ellas son: Born Globals (Knight y Cavusgil, 1996), Global Start-ups (Oviatt y McDougall, 1995), High Technology Star-ups (Burgel y Murray, 2000), Global High-Tech Firms (Roberts y Senturia, 1996), Instant Internationals (Fillis, 200I), Micromultinational (Dimitratos et al., 2003) y las International New Ventures (Oviatt y McDougall, 1994).Aún cuando un análisis meticuloso permite encontrar diferencias entre ellas, para efectos prácticos, se trabajará bajo el concepto de International New Ventures (INV).

Este trabajo pretende aportar a esta última corriente de pensamiento estudiando el comportamiento de las pequeñas y medianas empresas exportadoras (Pymex) en Chile. Los resultados que arroja esta investigación respaldan parcialmente la teoría de las INV. Para el caso chileno fueron estudiadas las variables sugeridas por Oviatt y McDougall (2005): redes de apoyo, nivel tecnológico de la industria en que participa la empresa, experiencia previa, tamaño de la empresa y las distancias psíquicas entre Chile y los países a los que se exporta.
Es importante señalar que la economía chilena presenta altos costos para la internacionalización. Esto se debe, entre otras cosas, a su ubicación geográfica. Sin embargo, al igual que otros estudios hechos en Estados Unidos, Australia, Dinamarca, Suecia, Nueva Zelanda y España, la experiencia previa se considera un factor determinante que poseen los emprendedores para una internacionalización acelerada. Otro aspecto que se destaca de las INV chilenas es que se caracterizan por una creciente tendencia a exportar a países geográfica y culturalmente lejanos. Sin embargo, estos resultados exploratorios para el caso de Chile revelan que el nivel tecnológico y las redes de apoyo no resultan ser tan influyentes como en otras economías.

Después de esta introducción, el trabajo se desarrolla de la siguiente manera: en la próxima sección se da un marco general sobre los procesos de internacionalización en pymex. La sección 3 plantea las hipótesis de estudio. En la sección 4 se discute la metodología de estudio. La sección 5 está dedicada a la presentación de los resultados y se concluye con recomendaciones para políticas públicas a partir de las implicancias de este estudio.

\section{Procesos de Internacionalización de Pymes}

Existen principalmente dos vertientes teóricas que pretenden explicar el actuar de las pymes respecto a su proceso de internacionalización. Éstas son el enfoque de Uppsala, que habla de un proceso gradual, y la otra corriente de internacionalización acelerada es el enfoque de las llamadas International New Ventures.

\section{Procesos Graduales}

El enfoque de Uppsala entiende el proceso de internacionalización como una etapa más dentro de la vida de las empresas que aspiran ingresar a mercados externos. El modelo concibe la internacionalización como un proceso gradual en que las empresas exportan de forma pausada a países donde las distancias psíquicas ${ }^{\mathrm{a}}$ son menores.

Según esta teoría existe un patrón que describe a las empresas que buscan internacionalizarse. En sus orígenes ellas sólo atenderán el mercado local, para luego comenzar con exportaciones de forma irregular. Luego, se sigue con agentes independientes como vía para exportar. Cuando ya se ha

aLas distancias psíquicas son el conjunto de impedimentos que sienten los inversionistas al momento de exportar a diversos países. Estas barreras pueden deberse a diferencias culturales, distancias geográficas, diferencias institucionales o ausencia de acuerdos comerciales que faciliten el intercambio comercial. 
adquirido el conocimiento suficiente, se establecen filiales comerciales y el proceso de internacionalización finaliza con establecer una filial productiva.

En esencia este modelo nos dice que el proceso de internacionalización es un proceso evolutivo, donde las pymes se vuelven cada vez más internacionales a medida que pasa el tiempo. El progresivo aumento del conocimiento y de la experiencia permiten superar las barreras psicológicas y reducir los riesgos asociados a una internacionalización profunda.

Este modelo está respaldado por una serie de investigaciones Johanson y Wiedershein, 1975; Johanson y Vahlne, 1977; Vahlne y Nordström, 1993). Sin embargo, también ha sido criticado por otros (Turnbull, 1987; Hamill, 1997), quienes argumentan que no representa el actuar de las pymes en el contexto actual. Desde que se desarrolló el modelo gradual (década del 70) hasta la fecha, han surgido una serie de cambios en el entorno económico, tales como el desarrollo de nuevas formas de negocio (ej: e-commerce), avances en las tecnologías que permiten una mayor eficiencia en los tiempos de traslado, desarrollo en las telecomunicaciones, reducción a las barreras de comercio, etc.

\section{Procesos Acelerados}

Dada la globalización de los negocios, los aumentos en la competencia, las alianzas estratégicas y los desarrollos en los sistemas de información, entre otros, es que existe la necesidad por parte de las empresas de internacionalizarse de manera más temprana y rápida que en el pasado.

La teoría de las International New Ventures explica que las pymes no necesariamente siguen un procedimiento gradual de internacionalización, sino más bien, gracias a características que posee el fundador -como las redes de contactos, alianzas, conocimientos previos, entre otros- las empresas comienzan una internacionalización inmediata concentrando una gran cantidad de esfuerzo y recursos (Oviatt y McDougall, 2005).

Las empresas, según este modelo, se caracterizan por desarrollar ventajas competitivas a través del aprovechamiento de los recursos disponibles para exportar a una serie de países. Estas empresas buscan crecer a través de las ventas en los mercados externos, enfocándose en atender, principalmente, a nichos de mercado (Oviatt y McDougall, 1994).

Es posible identificar ciertas características particulares en este tipo de empresas, como el rol del fundador y sus características emprendedoras (Madsen y Servais, 1997; Harveston et al, 200I). A su vez, son empresas mayormente propensas a estar en industrias de alto nivel tecnológico y que tienden a tener una base tecnológica (Knight y Cavusgil, 1996). En otras investigaciones se nombra la presencia de capital extranjero dentro de una empresa como factor clave para su internacionalización (Crick y Jones, 2000). Asimismo, se destacan las redes personales y corporativas con agentes públicos y privados como elementos para conseguir nuevos recursos, es decir, empresas que logran una eficiente utilización de factores tecnológicos y no tecnológicos (Holmlund y Kock, 1998).

Dadas estas características existen una serie de criterios para definir a las International New Ventures (Autio et al., 2000; Rennie, 1993; McDougall et al., 1994; Chetty y Campbell-Hunt; 2004). Para esta investigación fueron usadas las definiciones de Knight et al. (2004) y Madsen et al. (2000). De esta manera, se han definido a las INV como empresas que previo al tercer año desde su fundación exportan cifras mayores al $25 \%$ del total de sus ventas.

\section{Hipótesis de Estudio}

Para analizar el comportamiento de las pequeñas y medianas empresas exportadoras (pymex) chilenas se definieron una serie de variables que según la literatura afectan la conducta de las empresas. Dentro de los posibles determinantes de la internacionalización acelerada se analizan las redes de contacto, el apoyo institucional, nivel tecnológico, distancias psíquicas, tamaño de la empresa, apoyo institucional y la experiencia previa del emprendedor.

\section{Influencia de las redes de apoyo}

La influencia de las redes es una poderosa herramienta para emprendedores y el desarrollo de sus negocios (Dubini y Aldrich, 199I). McDougall et al. (1994) señalan que las redes permiten identificar más fácilmente oportunidades de negocios, y reducen el efecto de las distancias psíquicas. Las alianzas podrían generar también aportes en el know-how sobre los procesos productivos o comerciales. Por otra parte, ayudan a las compañías a incrementar su innovación al facilitar el acceso de los conocimientos de sus compañeros y complementar fuerzas a través de la creación de sinergias. La transferencia de conocimientos junto con los contactos internacionales, podrían facilitar el acceso a nuevos mercados y el descubrimiento de oportunidades.

Cabe señalar que un emprendedor puede tener diferentes tipos de redes o contactos internacionales. Aldrich (1999) señala que hay lazos fuertes y lazos débiles. Por una parte, un vínculo fuerte se caracteriza por una relación duradera, basada en confianza y enfocada en la generación de una alianza estratégica. Una forma de contacto internacional basado en un vínculo fuerte es a través de aportes de capital extranjero dentro del capital inicial de las empresas. Por lo tanto:

ISSN: 07 I8-2724. (http://www.jotmi.org)

Journal of Technology Management \& Innovation (c) Universidad Alberto Hurtado, Facultad de Economía y Negocios. 
Hipótesis I: La presencia de capital extranjero en el capital inicial de una pymex incrementa la probabilidad de que ésta sea INV.

Por otra parte, están los vínculos débiles, que se generan por la relación con clientes, proveedores e incluso amistades (Aldrich, 1999). Si bien este tipo de lazos no es tan estrecho como el primero, sigue entregando importantes beneficios para los emprendedores. La principal ventaja de este tipo de lazos radica en el hecho que, al requerir menos inversión que los lazos fuertes, son más numerosos (Oviatt y McDougall, 2005). De esta manera, una forma que puede tomar un lazo débil puede ser el pertenecer a alguna organización gremial, asociación o el apoyo de instituciones gubernamentales. El apoyo brindado por éstas puede facilitar a las empresas a comenzar su proceso internacionalización. La participación de empresas en este tipo de organizaciones se puede entender como un complemento, o sustituto en caso de ausencia, a las redes de contacto desarrolladas por el empresario fundador. Con la ayuda de ellas se logra una mayor eficiencia en los trámites y procesos para exportar. Por lo tanto:

Hipótesis 2: La presencia en asociaciones, gremios u otro tipo de institución aumenta la probabilidad de que la pymex sea INV.

\section{Nivel Tecnológico}

Knight y Cavusgil (1996) definen a las born global como compañías pequeñas de orientación tecnológica que participan en mercados internacionales desde los primeros días de su creación. Es así que el estudio del nivel tecnológico ha sido incluido desde los orígenes del estudio del comportamiento pymes (Oviatt y McDougall, 1995; Knight y Cavusgil, 1996; Harveston et al., 200I).

Adicionalmente, Grégoire et al. (2008) señalan que uno de los factores más trascendentales para las empresas que en etapas iniciales se internacionalizan es ver factores propios de la industria y la relación que existe entre el riesgo y retorno de esta decisión. Las empresas en sectores tecnológicos altos están envueltas en mercados de constante cambio y las ventanas de oportunidades de crecimiento no están abiertas por mucho tiempo (Crick y Spence, 2005). En ese sentido, limitarse a satisfacer sólo el mercado local no sería una estrategia muy favorable y la internacionalización permitiría extender el periodo en que las ventanas de oportunidades están abiertas (Karagozoglu y Lindell, 1998). Por lo tanto:

Hipótesis 3: el mayor nivel tecnológico del sector en que participe la pymex aumenta la probabilidad de que sea INV.

\section{Distancias psíquicas}

Las International New Ventures se caracterizan por poseer una amplia red de contactos que les permite detectar oportunidades en mercados internacionales. McDougall et al. (1994) señala que las redes de contacto tienen mayor influencia que las distancias psíquicas. Este concepto se refiere a las diferencias que existe entre distintos países, donde algunas de las principales razones responden a factores culturales y geográficos (Johanson y Vahlne, 1977; Stottinger y Schlegelmilch, 1998). De acuerdo a las teorías de las INV, estas empresas se caracterizan porque aún a costa de las diferencias en términos culturales están dispuestas a explotar las oportunidades en mercados lejanos, pero altamente atractivos.

Siguiendo con la misma lógica anterior, las empresas no debieran prestar atención a las distancias geográficas -que es uno de los componentes de las distancias psíquicas- mientras exista la oportunidad de capturar mercados internacionales al poseer las redes de contacto necesarias. El desarrollo de las tecnologías de la información ha podido suplir en gran medida las dificultades de estar presente en mercados lejanos geográficamente. Herramientas como Internet permiten a las personas estar ubicables en prácticamente cualquier parte del mundo. Knight y Cavusgil (1996) señalan que para las born global resulta irrelevante el concepto de distancias psíquicas en su decisión de internacionalización. Debido a que en Chile los mercados más atractivos se encuentran lejanos geográficamente y en términos culturales son diferentes al contexto chileno, es probable que se intensifiquen las diferencias entre las empresas que siguen un patrón de internacionalización gradual de las INV. Por lo tanto:

Hipótesis 4: las INV chilenas se caracterizan por exportar a mercados psicológicamente más lejanos que las empresas de internacionalización gradual.

\section{Tamaño}

Al igual que en el caso del nivel tecnológico, el tamaño de la empresa es una variable estudiada desde los comienzos de las investigaciones sobre internacionalización de pymes, principalmente debido a que el tamaño inicial que tienen las empresas afecta la estrategia de éstas (BarNir et al., 2003; Gabrielsson et al., 2008). Las empresas de menor tamaño tienden a tener mayores dificultades para crear ventajas competitivas por su incapacidad de generar economías de escalas, por otra parte tienden a tener dificultades para hacer un buen reconocimiento del mercado y son escasos los recursos para asegurar un buen posicionamiento (Shamsie et al., 2004). 
Por otra parte, en general, las empresas de mayor tamaño poseen más recursos organizacionales, mejores capacidades directivas y una mayor adaptabilidad para manejarse en entornos complejos y en constantes cambios (Chung et al., 2007). La presencia de trabajadores, sobre todo de aquellos con experiencias previas en temas de exportación, debiera permitir una acumulación de activos intangibles muy valiosos para que una empresa internacionalice sus actividades. Por lo tanto:

Hipótesis 5: a mayor tamaño de una empresa, se incrementa la probabilidad de que una pymex sea INV.

\section{Experiencia}

En prácticamente la totalidad de las corrientes teóricas de gestión se reconoce que las características propias del emprendedor cumplen un rol determinante en la estrategia del negocio. De hecho, la generación del emprendimiento está basado en la capacidad del emprendedor de reconocer oportunidades de negocios (Shane y Venkataraman, 2000). En este sentido, existen dos factores determinantes para la creación de nuevos negocios. Por un lado están las oportunidades de negocios y por el otro, personas que sean capaces de reconocerlas y saber explotarlas.

Oviatt y McDougall (2005) considera que uno de los principales aspectos que acelera la internacionalización de empresas es el emprendedor, ya sea por sus rasgos psicológicos (por ejemplo la aversión al riesgo) y sus características personales (por ejemplo conocimiento basado en experiencias previas). La percepción de los emprendedores sobre las oportunidades de negocio se debiese ver reflejada en la estrategia de internacionalización, donde las empresas que cuenten con personas que posean el know-how de cómo exportar, sean las que también aceleren su proceso de internacionalización. Por lo tanto:

Hipótesis 6: Empresas con un fundador que posee experiencia previa en internacionalización de negocios aumenta la probabilidad de que ésta sea INV.

\section{Metodología de estudio}

\section{Selección de la muestra}

De acuerdo a las hipótesis anteriores, utilizaremos una muestra de II 2 pymex de Chile, a la que se le aplicará un modelo de regresión logística para estudiar el cambio en la probabilidad de que una joven empresa exportadora sea clasificada como INV. La muestra de I I 2 empresas fue construida utilizando una base de datos de Pymexporta \{http:// www.pymexporta.cl/pymexporta/fontcolorConvocatoriasanterioresfont/tabid/2986/Default.aspx\}. Teniendo el nom- bre de las empresas, parte de la información usada para este estudio es pública y la información adicional que se necesitaba fue recolectada a través de una encuesta respondida telefónicamente o vía correo electrónico. La base de datos quedó compuesta por 43 empresas clasificadas como INV (según el criterio desarrollado por Knight et al. (2004) y Madsen et al. (2000) y 69 empresas que siguen un proceso gradual de internacionalización.

En relación a las empresas, es importante mencionar que una fracción no menor pertenece al rubro agrícola y gran parte de sus trabajadores no son de tiempo completo, sino sólo contratados por periodos de tiempo definidos y para la realización de trabajos que no requieren un capital humano muy alto, por el caso de los temporeros. Por otra parte, Chile es una economía donde los principales productos exportados corresponden a materias primas y productos de bajo valor agregado, dentro de los que se destaca el vino, maderas, frutas y pescados.

El detalle de las exportaciones realizadas, monto exportado y países destinos fue posible obtener de la página de la Dirección de Promoción de Exportaciones (www.prochile.cl).

\section{Variables de estudio}

La variable dependiente muestra la probabilidad de que una joven empresa exportadora siga el patrón exportador de las INV. Mientras más cercano a I sea el valor que tome la variable dependiente, mayor será la probabilidad de ser clasificada como INV. Análogamente, mientras más cercano a 0 sea el valor de la variable dependiente, mayor será la probabilidad de ser clasificada como una empresa de internacionalización gradual.

Las variables independientes utilizadas para este estudio son: alianzas internacionales, redes, nivel tecnológico del sector en que participa la empresa, distancia psíquica, tamaño de la empresa y experiencia. El detalle para cada variable es el siguiente:

(a) Alianzas internacionales: variable binaria que indica si dentro del capital de la empresa existen aportes que provienen de fuentes internacionales. La variable adquiere valor I cuando existe aportes de capital extranjero y 0 cuando no existe ningún tipo de aporte.

(b) Redes de apoyo: variable binaria que señala la afiliación de la empresa con alguna institución como guía para exportar, tomando en dicho caso el valor I. En caso de ausencia de alguna institución como guía para exportar, el indicador toma valor 0 .

(c) Nivel tecnológico: variable binaria que indica si la 
empresa participa en un sector tecnológico alto o bajo. La definición utilizada sobre los niveles tecnológicos por sector productivo fue la diseñada por la organización para la cooperación y el desarrollo económico (OCDE). En caso de que el rubro de la empresa sea considerado de alto nivel tecnológico, el indicador toma valor I. Por el contrario, si la empresa participa en una industria de bajo nivel tecnológico, el indicador toma valor 0 .

(d) Distancia psíquica: variable que explica el porcentaje de países clientes, en función de las cantidades exportadas, que presentan una baja distancia psíquica respecto al total de países a los cuales una pyme exporta. La forma para determinar la distancia psíquica fue a través del idioma oficial de cada país y de la ubicación geográfica. Mientras más cercano a I sea el valor, significa que no existen mayores distancias psíquicas, en términos de kilómetros (distancia geográfica) e idiomáticas (distancia cultural), con los países a los cuales exporta.

(e) Tamaño de la empresa: variable cuantitativa que se creó según el número de empleados de la empresa. Se clasificaron de acuerdo a tres rangos, siendo el primer rango empresas que tengan entre $\mathrm{I}$ a 50 empleados, el segundo rango son las pymex que tienen entre $5 \mathrm{I}$ a 100 empleados $y$ el tercer rango son para aquellas que tienen valores superiores a los 101 empleados.

(f) Experiencia: variable binaria que indica si el em- prendedor posee conocimientos previos sobre el proceso de internacionalización de negocios. La variable toma valor I cuando sí existe experiencia previa en negocios internacionales. En caso de no existir, la variable toma valor 0 .

Con el objetivo de acentuar el estudio al contexto chileno, se ha incorporado un aspecto adicional que haga mención a la apertura comercial que posee Chile. Es así que, como variable de control, hemos adicionado un índice que va desde cero a uno y representa el porcentaje de países de destino donde Chile posee algún tipo de tratado comercial (como por ejemplo TLC). Cada país de destino que sí posee un acuerdo comercial con Chile posee valor I, en aquellos países donde no existe ningún acuerdo comercial el valor es 0 . Luego, para cada empresa se calculó el porcentaje países con acuerdos comerciales respecto a su total de países de destino.

La Tabla I muestra la matriz de correlaciones entre las variables del modelo. En ésta se puede observar cómo el tamaño es la única de las variables que presenta una relación inversa, aunque no significativa, con la probabilidad de ser una INV. El resto de las variables presentan una relación directa, destacándose las distancias psíquicas y la experiencia previa, que son significativas al $95 \%$. Por otra parte, el apoyo de redes a través del capital de la empresa como la participación en algún tipo de gremio o asociación como guía para exportar, también presentan una relación estadísticamente significativa al $90 \%$.

\begin{tabular}{|c|c|c|c|c|c|c|c|c|c|c|c|}
\hline & & Media & D.E. & 1 & 2 & 3 & 4 & 5 & 6 & 7 & 8 \\
\hline 1 & INV & 0,38 & 0,489 & 1,000 & & & & & & & \\
\hline 2 & $\begin{array}{l}\text { Capital internac- } \\
\text { ional }\end{array}$ & 0,23 & 0,424 & $0,218^{*}$ & 1,000 & & & & & & \\
\hline 3 & $\begin{array}{l}\text { Industria de alto } \\
\text { nivel tecnológico }\end{array}$ & 0,08 & 0,273 & 0,037 & 0,071 & 1,000 & & & & & \\
\hline 4 & Distancia psíquica & 0,63 & 0,338 & $0,300^{* *}$ & 0,011 & $-0,191^{*}$ & 1,000 & & & & \\
\hline 5 & $\begin{array}{l}\text { Tamaño de la } \\
\text { empresa }\end{array}$ & 1,62 & 0,819 & $-0,011$ & 0,077 & $-0,143$ & $-0,154$ & 1,000 & & & \\
\hline 6 & $\begin{array}{l}\text { Distancia institu- } \\
\text { cional }\end{array}$ & 0,03 & 0,076 & 0,144 & 0,039 & 0,068 & 0,129 & 0,131 & 1,000 & & \\
\hline 7 & Asociaciones & 0,46 & 0,500 & $0,237^{*}$ & $0,347^{* *}$ & $-0,006$ & $-0,007$ & 0,079 & 0,132 & 1,000 & \\
\hline 8 & $\begin{array}{l}\text { Experiencia pre- } \\
\text { via }\end{array}$ & 0,55 & 0,499 & $0,450^{* *}$ & $0,366^{* *}$ & 0,133 & $-0,016$ & 0,106 & 0,172 & $0,460^{* *}$ & 1,000 \\
\hline
\end{tabular}

Tabla I: Matriz de correlaciones * = Estadísticamente significativo al $90 \%$ **: Estadísticamente significativo al $95 \%$

ISSN: 07I 8-2724. (http://www.jotmi.org)

Journal of Technology Management \& Innovation (c) Universidad Alberto Hurtado, Facultad de Economía y Negocios. 


\section{Resultados del Modelo}

De acuerdo a los resultados expuestos en la Tabla 2, las variables asociadas al emprendedor son las únicas que presentan valores estadísticamente significativos. Estos son: distancias psíquicas percibidas y experiencia previa en internacionalización.

La variable distancia psíquica hace referencia a la percepción que tiene el dueño sobre la distancia de los mercados de destino, ya sea por temas geográficos o culturales. Este aspecto es un factor que determinaría la velocidad en que las empresas para ir en búsqueda de destinos internacionales. A pesar de la distancia y las diferencias que puedan existir, las INV estarían dispuestas a conquistar mercados altamente atractivos. Debido a que éstos son en su mayoría distantes, las empresas que siguen el patrón de las INV deben recorrer grandes distancias para participar en ellos. La velocidad de internacionalización es esencial, puesto que el satisfacer primero a un determinado mercado dejaría a la firma en una mejor posición frente a sus competidores. De esta manera, es posible reconocer cierta diferencia sobre las empresas de internacionalización gradual, quienes sí prestarín atención a las distancias psíquicas.

Así también los resultados indicarían que poseer experiencia previa en el proceso de internacionalización sería determinante para acelerar la estrategia de expansión. Las empresas chilenas que siguen un proceso acelerado de internacionalización se caracterizan por poseer conocimientos previos sobre exportación. En este sentido, es posible señalar que la estrategia de internacionalización de las pymex depende fuertemente de las habilidades, percepciones y conocimientos propios del dueño.

La variable tamaño no resultó ser significativa. Esto implicaría que la acumulación de capital humano no necesariamente convergerá en una estrategia de internacionalización acelerada. Respecto a esta variable un factor importante, y que puede ayudar a entender el resultado es el hecho de que, así como fue mencionado, las empresas encuestadas contrataban temporeros para los tiempos de cosecha y el resto de la temporada permanecía sólo el personal administrativo. Éste último, dentro de las empresas encuestadas, resultó ser en general muy reducido (no superando los dos dígitos).

A diferencia de lo observado en otros estudios de las INV en otros contextos, las redes de contacto en Chile no estarían cumpliendo un rol tan fundamental para acelerar la velocidad en que se desarrolla la estrategia de internacionalización. La ausencia de algún tipo de redes, ya sea a través de alianzas con empresas en el extranjero, o través de alguna institución encargada de facilitar el intercambio, no estaría necesariamente apresurando o retrasando la expansión hacia otros mercados.

La variable nivel tecnológico tampoco resultó ser determinante, a diferencia de lo que señala la teoría de las INV. Esto posiblemente se deba a las características propias de la región y sus ventajas comparativas frente al resto del mundo. Chile se caracteriza por poseer un suelo y clima favorable para el cultivo de una gran variedad de hortalizas. De hecho, en Chile la mayor cantidad de exportaciones se encuentra en vinos con denominación de origen. Otros estudios en

\begin{tabular}{lrrrrrrr}
\hline & B & E.T. & \multicolumn{1}{l}{ Wald } & gl & Sig. & Exp(B) \\
& & & & & & \\
Redes 1: Capital extranjero & 0,220 & 0,588 & 0,140 & 1 & 0,708 & 1,246 \\
Redes 2: Asociaciones & 0,216 & 0,542 & 0,158 & 1 & 0,691 & 1,241 \\
Nivel tecnológico & 0,797 & 0,870 & 0,840 & 1 & 0,359 & 2,219 \\
Distancia psíquica & 3,096 & 0,931 & 11,047 & 1 & 0,001 & 22,104 \\
Tamaño de la empresa & 0,049 & 0,302 & 0,026 & 1 & 0,872 & 1,050 \\
Experiencia previa & 2,334 & 0,608 & 14,755 & 1 & 0,000 & 10,323 \\
Acuerdos comerciales & 0,464 & 3,382 & 0,019 & 1 & 0,891 & 1,591 \\
Constante & $-4,307$ & 1,101 & 15,308 & & 1 & 0,000 & 0,013 \\
Estadísticos & & & & & & \\
Número de casos & 112 & & & & & \\
R2 Nagelkerke & 0,410 & & & & & \\
R2 Cox y Snell & 0,302 & & & & & \\
\hline
\end{tabular}

Tabla 2: resultados del modelo

ISSN: 07 I8-2724. (http://www.jotmi.org)

Journal of Technology Management \& Innovation (c) Universidad Alberto Hurtado, Facultad de Economía y Negocios. 
países donde hay acceso a la tecnología a un menor costo, como España, este factor sí resulta significativo. Por lo tanto, sería razonable suponer que en un país donde gran parte de la fuerza exportadora está basada en commodities, $y$ en productos de bajo nivel agregado, el nivel tecnológico no sea un aspecto fundamental para la velocidad de internacionalización.

En relación a la variable que mide los destinos comerciales de las pymex chilenas según los acuerdos que existen entre países tampoco resultó ser estadísticamente significativa. Lo anterior podría deberse a la característica propia del país, el cual es una de las economías más abiertas del mundo. El hecho de poseer acuerdos comerciales que simplifiquen las transacciones entre países si bien es importante, no tendría necesariamente un impacto en la elección de las empresas para desarrollar una expansión internacional de forma acelerada o gradual.

\section{Conclusión e implicancias}

El cumplimiento de las teorías de internacionalización acelerada para el caso chileno estaría restringido por las características propias de este país. Pese a que Chile se caracteriza por ser uno de los países con más acuerdos comerciales del mundo, este factor no determinaría la estrategia de internacionalización para las pymex. Sin embargo, debido a que su ubicación geográfica es distante de las principales economías del mundo, la búsqueda de mercados altamente atractivos induce a las INV a no tomar en cuenta estas barreras, mientras puedan capturar mercados donde la relación de riesgo/retorno sea mejor.

De acuerdo a los resultados de este estudio, las pymex chilenas estarían presentando una alta centralización en la toma de decisiones. En este estudio fueron analizadas dos formas de redes, una para lazos fuertes y otra para lazos débiles. Sin embargo, ambas formas de testeo para las redes de apoyo -reconociéndolas como factores claves para facilitar la apertura hacia mercados extranjeros- no serían herramientas que afecten la velocidad de internacionalización.

Dado que, así como fue mencionado, en la mayoría de los casos de la muestra seleccionada la mano de obra era no calificada, no es posible afirmar si realmente la acumulación de capital en la empresa resulta ser un aporte a la estrategia de internacionalización. Sin embargo, sí fue posible constatar que en empresas donde el dueño poseía experiencia previa en negocios internacionales sí habría un efecto positivo sobre el patrón exportador. Así también, adicionalmente a la experiencia, la búsqueda de mercados atractivos, pese a las distancias psíquicas que podrían existir, sí sería una características de las INV chilenas. Por lo tanto, en ambos casos estamos hablando de características individuales del dueño y su influencia en la toma de decisiones estratégicas de la empresa. En este sentido, una política pública que esté enfocada directamente en potenciar las habilidades y conocimientos propios del emprendedor sería una buena herramienta para fomentar el desarrollo de las INV.

El fortalecimiento del capital humano que posean los emprendedores debe considerarse como una herramienta complementaria de política pública a las actuales medidas que se han desarrollado. En un mercado como el chileno donde existen grandes barreras de entrada para los nuevos emprendedores y la apertura del mercado interno es baja (Amorós y Poblete, 2012), es importante el desarrollo de políticas que permiten a las nuevas empresas ir en búsqueda de mercados internacionales desde sus inicios.

Hasta la fecha, los esfuerzos en política pública se han enfocado en temas más institucionales, elaboración de tratados comerciales, desarrollo y fortalecimiento de asociaciones. Los resultados de este estudio indican que para fomentar una internacionalización acelerada más que concentrarse en temas institucionales, que son fundamentalmente importantes, las características propias del emprendedor es la principal herramienta que afecta la estrategia de las empresas para ir en búsqueda de mercados extranjeros. En este sentido, políticas enfocadas en aumentar el capital humano del emprendedor sería una buena forma de fomentar a las pymex para que éstas desarrollen una estrategia de internacionalización acelerada.

\section{Limitaciones del estudio}

Los resultados obtenidos de esta investigación pueden estar limitados por las características específicas con las cuales fueron medidas cada una de las variables, así también por la muestra con la que se realizó este estudio. Dado lo anterior está la posibilidad que en una eventual realización de la misma investigación usando otros proxys los resultados difieran a los expuestos en este trabajo. 


\section{Referencias}

ALDRICH, H. (1999): Organizations evolving. London: Sage Publications.

AUTIO, E., Sapienza, H.J., y Almeida, J.G. (2000): “Effects of age at entry, knowledge intensity, and imitability on international growth.Academy of Management Journal, 43, 909-924.

AMORÓS,J. E. y Poblete, C. (2012): Global Entrepreneurship Monitor, 20II Reporte Nacional de Chile 20II. Santiago: Ediciones Universidad del Desarrollo.

BARNIR, A., Gallaugher, J. y Auger, P. (2003): "Business process digitization, strategy, and the impact of firm age and size: the case of the magazine publishing industry". Journal of Business Venturing, |8(6), 789-8|4.

BURGEL, O. y Murray, G.C. (2000): "The Effect of Internationalization on the Rate of Growth of High-Tech StartUps". Frontiers of Entrepreneurial Research 2000, Babson College M.A.

CHETTY, S. y Campbell-Hunt, C. (2004): "A Strategic Approach to Internationalization: A traditional Versus a BornGlobal Approach". Journal of International Marketing, 12/I:57-8I.

CHUNG, H. -J., Chen, C. -C., y Hsieh, T. -J. (2008): "First geographic expansion of start-up firms: Initial size and entry timing effects". Journal of Business Research, 60(4), 388-395.

CRICK, D. y Jones, M. (2000): "Small High-Technology Firms and International High-Technology Markets". Journal of International Marketing, 8 (2), 63-85.

CRICK, D. y Spence, M. (2005): "The internationalisation of 'high performing' UK high-tech SMEs: a study of planned and unplanned strategies". International Business Review, I4(2), 167-185.

DIMITRATOS, P., Johnson, J.E., Slow, J y Young, S. (2003): “Micromultinationals: New Types of Firms for the Global Competitive Landscape", European Management Journal, 2I(2), 164-174.

DUBINI, P.y Aldrich, H. (|99|): "Personal and extended networks are central to the entrepreneurial process". Journal of Business Venturing, 6, 305-3163.

FILLIS, I. (200I): "Small firm internationalization: An investigative survey and future research directions". Management Decision, 39/9, 767-783.
GABRIELSSON, M., Manek Kirpalani, V., Dimitratos, P., Solberg, C. y Zucchella, A. (2008): "Born globals: Propositions to help advance the theory". International Business Review. 17, 385-40|

GRÉGOIRE, D.,Williams, D y Oviatt, B. (2008): "Early internationalization decisions for new ventures: what matters?" Frontiers of Entrepreneurship Research, 28//8, 2.

HAMILL, J. (1997): The Internet and international marketing, International Marketing Review, I 4 (5), 300-323.

HARVESTON, P., Kedia, B., Davis, P. y Van Scotter, J. (200I): "Synoptic versus incremental internationalization: an examination of born global and gradual globalizing firms". Frontiers of Entrepreneurship Research, Babson Park. Wellesley (Mass).

HOLMLUND, M. y Kock, S. (1998): "Relationship and the internationalization of finish small-and-medium sized companies", International Small Business Journal, 46-63.

JOHANSON, J. y Vahlne, J. (1977): “The internationalization process of the firm :A model of knowledge development an increasing foreign market commitments". Journal of International Business Studies, volumen 8, II-24.

JOHANSON, J. y Vahlne, J. (1990): "The mechanism of Internationalization". International Marketing Review, 7, 4, I I-34.

JOHANSON, J. y Wiedershein, P. (1975): "The internationalization Process of the Firms: Four Swedish Case Studies". Journal of Management Studies, 305-322.

KARAGOZOGLU, N., y Lindell, M. (1998): "Internationalisation of small and medium-sized technology-based firms: an exploratory study". Journal of Small Business Management, 36(I), 44-59.

KNIGHT, G. y Cavusgil, S. (1996): "The born global firm: a challenge to traditional internationalization theory".Advances in International Marketing, JAI Press, I I-26.

KNIGHT, G., Madsen, T. K., y Servais, P. (2004): “An inquiry into born-global firms in Europe and the USA". International Marketing Review, 2 I (6): 645-665.

MADSEN, T. K. y Servais, P. (1997): “The Internationalization of Born Globals: An Evolutionary Process?". International Business. Review, volumen 6, 6,56I - 583. 
MADSEN, T.K., Rasmussen, E.S. y Servais, P. (2000): Differences and similarities between born globals and other types of exporters. In A. Yaprak \& J. Tutek (eds.) Globalization, The Multinational Firm, and Emerging Economies.Advances in International Marketing,

AMSTERDAM: JAI/Elsevier Inc., 247-265.

MCDOUGALL, P., Shane, S. y Oviatt, B. (1994): “Explaining The Formation Of International New Ventures: The Limits Of Theories From International. Business Research". Journal of Business Venturing, 9, 469-487.

OVIATT, B. y McDougall, P. (1994): "Toward a Theory of International New Ventures". Journal of International Business Studies, 25, I: 45-64.

OVIATT, B. y McDougall, P. (1995): “Global Start-ups: Entrepreneurs on a Worldwide Stage". Academy of Management Executive, 9, 2:30-43.

OVIATT, B. y McDougall, P. (2005): “Defining International Entrepreneurship and Modeling the Speed of Internationalization". Entrepreneurship Theory and Practice. 537-553.

RENNIE, M. (1993): “Global competitiveness: Born global”. The McKinsey Quarterly, 4, 45-52.

SHAMSIE, J., Phelps, C. y Kuperman J. (2004): "Better late than never: a study of late entrants in household electrical equipment". Strategic Management Journal, 25, 69-84.

SHANE, S. y Venkataraman, S. (2000): "The promise of entrepreneurship as a field of research". Academy of Management Review 25(I):217-226.

STOTTINGER, B. y Schlegelmilch, B. (1998): "Explaining export development through psychic distance: enlightening or elusive". International Marketing Review, 15 (5), 357-372.

TURNBULL, P. (1987): "A challenge to the stages theory of the internationalization process". En Rosson, P. y Reid, S. (eds): Managing export entry and expansion, 2I-40.

ROBERTS, E. y Senturia, T.(1996): "Globalizing the Emerging High-Technology Company”. Industrial Marketing Management, 25: 49I-506

VAHLNE J. y Nordström, K. (1993): "The internationalization process: impact of competition and experience". International Trade Journal, volumen 7, 529-548. 